\title{
La Pedagogía como Ciencia para el Tratamiento de los Contenidos Generales del Proceso Educativo y la Formación de Valores
}

\author{
José M. Ramos, Bertha S. Rhea, Ramón V. Pla y Omar Abreu \\ Universidad Técnica del Norte, Campus. Avenida 17 de julio, 5-21, General José María Córdova, Código \\ postal 199, Ibarra, Ecuador, (e-mail: Joseramosbanobre@sma.unica.cu, srheag@utn.edu.ec \\ rplalopez@sma.unica.cu, oabreu@utn.edu.ec)
}

Recibido May. 24, 2017; Aceptado Jul. 25, 2017; Versión final Ago. 9, 2017, Publicado Dic. 2017

\begin{abstract}
Resumen
El objetivo del estudio que se presenta es explicar las potencialidades de la Pedagogía como ciencia para el tratamiento de los contenidos generales y la formación de valores en el proceso educativo incluidos en la malla curricular de las carreras universitarias. Se utilizó el método de ascenso de lo abstracto a lo concreto, inductivo-deductivo histórico lógico y analítico sintético, sistémico, dialéctico, y la metodología de la comparación por pares del criterio de expertos. Se definen los contenidos generales y se diferencian de los contenidos específicos establecidos en las asignaturas cuyo tratamiento le corresponde a la didáctica. Se definen y caracterizan los procesos, educativo y de enseñanza-aprendizaje, se explica la dialéctica entre ellos, se establecen los pasos metodológicos para el tratamiento pedagógico de los contenidos generales y se revelan posibilidades de estos procesos para la formación de valores, seleccionados y muy demandados en la sociedad actual.
\end{abstract}

\section{Pedagogy as a Science for the Treatment of the General Contents of the Educational Process and the Formation of Values}

\begin{abstract}
The objective of the study presented in this article is explaining the potential of Pedagogy as a science for the treatment of the general contents and the formation of values in the educational process included in the curricular program of university careers. The method of ascent from the abstract to the concrete, inductive-deductive logical historical and analytical synthetic, systemic, dialectical, and the methodology of peer comparison of the criterion of experts, was used. The general contents are defined and differentiated from the specific contents established in the subjects whose treatment corresponds to the didactics. The processes are defined and characterized, educational and teaching-learning, the dialectic between them is explained, the methodological steps for the pedagogical treatment of general contents are established, and the possibilities of these processes for the formation of selected values, highly demanded in contemporary society.
\end{abstract}

Keywords: educational process; pedagogy; general contents; human values 


\section{INTRODUCCIÓN}

Para que sea útil y efectiva la educación del presente y el futuro, requiere superar enfoques centrados en la transmisión-recepción de conocimientos fragmentados y ha de sostener acciones que promuevan un aprendizaje desarrollador, vinculando dialécticamente a docentes, alumnos y a la comunidad en su conjunto, con propuestas de desarrollo personal y comunitario, desde todos los componentes y contextos del proceso educativo (PE). La educación debe concebirse como acción transformadora de la sociedad, asegurando el dominio de la cultura suficiente para formar individuos a tono con las necesidades de cada país. La educación es un proceso que involucra a todos los sectores de la sociedad y particularmente a la institución formativa y a la familia, frente al cual cada uno debe aportar su esfuerzo y capacidad. Las demandas de la formación de profesionales y la dinámica del mundo contemporáneo exigen niveles superiores de integración en la gestión educativa, sin embargo, aún se aprecian enfoques dogmáticos, que no centran su atención en la elevación de la calidad de la formación integral de la personalidad de los estudiantes, sino en el dominio de los contenidos de cada una de las asignaturas de la malla curricular vinculada al proceso de enseñanza-aprendizaje (PEA).

La categoría contenido se asocia generalmente a las asignaturas del PEA, pero existen contenidos generales y contextuales que trascienden las asignaturas y deben ser considerados en un proyecto educativo. La comunidad universitaria necesita de procedimientos para dar tratamiento a los contenidos generales en los proyectos educativos, los cuales se tratan hoy mediante las estrategias curriculares y dentro del PEA, lo que origina una confusión alrededor de los conceptos de Pedagogía, Didáctica, sus funciones, su objeto de estudio y su utilidad para el tratamiento de determinados contenidos o de todos los contenidos. También se confuinden términos como PE, PEA, proceso pedagógico y proceso formativo. Este tema ha sido incluido en la obra de autores como Abreu et., al (2017), Abreu et., al (2016) quienes opinan que estos procesos son distintos y que cada uno tiene su esencia propia y son objeto de estudio de ciencias diferentes. Pla et., al (2016), Pla et., al (2012) establecen diferencias entre el PE y el PEA, señalan que a la Pedagogía le corresponde el estudio del primero. Olivé y Finales (2015) también consideran que estos procesos no son lo mismo. Martín et., al (2013) hablan de la importancia del dominio del contenido y de la manera más efectiva de hacerlo. Gairin y Muñoz (2013) revelan la utilidad de la organización escolar como campo de estudio e investigación. Díaz (2009) analiza el conocimiento didáctico del contenido para la enseñanza. Bolívar (2005) analiza el vínculo contenido Didáctica. Fishbein y Azjen (2000) plantean los componentes del PE y sus relaciones con el desarrollo de las actitudes. Álvarez (1997) define la esencia del contenido y sus potencialidades. Álvarez (1999) dice que muchos autores identifican al PE como PEA y que esta posición es errónea. Gairin (2000) se ocupa de establecer los elementos necesarios para la relación entre los valores y sus características. Aranda et,. al (2005), Martínez (2006), Casares et,. al (2010), Osuna et., al (2012) y Vidal y Araña (2014), son autores que se refieren a la importancia de la formación de valores como punto esencial del desarrollo del PE. Los aportes teóricos de estos al tema de estudio se amplían en el resto del trabajo.

\section{METODOLOGÍA}

Se utilizaron el método de ascenso de lo abstracto a lo concreto para tomar como punto de partida la situación actual del tratamiento de los contenidos generales, inductivo-deductivo para determinar potencialidades y limitaciones del PE para el tratamiento de los contenidos generales desde la Pedagogía como ciencia, histórico lógico y analítico sintético para estudiar la historia de las definiciones de los procesos educativo, de enseñanza-aprendizaje, pedagógico y formativo y de la Pedagogía y la Didáctica, sistémico para diseñar los pasos metodológicos para el tratamiento de los contenidos generales, el dialéctico para establecer la relación de la Pedagogía con el PE y de la Didáctica con el PEA. Para la selección de los valores propuestos se utilizó la metodología de la comparación por pares del criterio de expertos. Se seleccionaron 15 expertos, 5 ecuatorianos, 5 cubanos, 1 boliviano, 2 españoles, 1 argentino y 1 colombiano. El promedio de experiencia profesional es de 17 años. Entre los expertos, 6 son Doctores en Ciencias Pedagógicas, el resto en otras especialidades, pero todas vinculadas a la docencia universitaria. Se les indicó que marcaran con una cruz (X), en una escala creciente de 1 a 10 , el valor que se corresponde con el grado de conocimiento e información acerca del tema objeto de investigación, considerando los indicadores siguientes: 1) participación en investigaciones relacionadas con el tema, 2) estudios teóricos relacionados con el tema, 3) participación en eventos internacionales relacionados con el tema y 5) nivel de conocimientos acerca del tema.

Tabla 1: Resultados de la autoevaluación de los expertos

\begin{tabular}{|c|c|c|c|c|c|c|c|c|c|}
\hline 1 & 2 & 3 & 4 & 5 & 6 & 7 & 8 & 9 & 10 \\
\hline 0 & 0 & 0 & 0 & 0 & 0 & 3 & 1 & 4 & 7 \\
\hline
\end{tabular}


A los expertos se les aplicó un cuestionario con las categorías muy adecuado (MA), bastante adecuado (BA), adecuado (A), poco adecuado (PA) o no adecuado (NA) a los siguientes indicadores: 1) vigencia del objeto de estudio, 1) planteamiento del objeto de estudio, 3) selección de los valores que pueden ser formados mediante el tratamiento de los contenidos generales, 4) utilidad de las definiciones de los valores y 5) potencialidades de la Pedagogía para el tratamiento de los contenidos generales y la formación de valores.

Tabla 2: Resultados de la evaluación de los expertos

\begin{tabular}{|c|l|l|l|l|l|c|c|}
\hline$N^{-}$ & \multicolumn{1}{|c|}{ Indicadores } & MA & BA & A & $P A$ & NA & Categoría definitiva \\
\hline 1 & Vigencia del objeto de estudio & 15 & & & & & MA \\
\hline 2 & Planteamiento del objeto de estudio & 15 & & & & & MA \\
\hline 3 & $\begin{array}{l}\text { Selección de los valores que pueden ser formados } \\
\text { mediante el tratamiento de los contenidos generales }\end{array}$ & 11 & 3 & 1 & & & BA \\
\hline 4 & Utilidad de las definiciones de los valores & 13 & 2 & & & & MA \\
\hline 5 & $\begin{array}{l}\text { Potencialidades de la Pedagogía para el tratamiento de } \\
\text { los contenidos generales y la formación de valores }\end{array}$ & 8 & 3 & 3 & 1 & & BA \\
\hline
\end{tabular}

\section{DISCUSIÓN Y RESULTADOS}

En la Pedagogía la relación de los objetivos y contenidos generales con los métodos, los medios, la evaluación y las formas organizativas, ha sido menos estudiada que en la Didáctica, respecto a los objetivos y contenidos de los programas docentes. Esto genera la tendencia al tratamiento similar de ambos tipos de contenidos y justifica que se subestime el carácter operativo de la Pedagogía y su sustitución por la Didáctica, cuando ambas tienen objetos y funciones diferentes. Estas problemáticas tienen implicación práctica, pero exige que se teorice acerca del tratamiento del componente contenido desde la Pedagogía como ciencia y que no sistematicen teorías de forma independiente sobre la educación física, ambiental, para la salud y la sexualidad, laboral, profesional, económica, patriótica, jurídica, moral, político-ideológica, estética, científico tecnológica, religiosa, y otras de acuerdo al contexto, sino logrando que la Pedagogía como ciencia ofrezca resultados teóricos y metodológicos para tratar los contenidos generales del PE.

\section{La Pedagogía y el proceso educativo}

En la teoría dedicada a la educación como proceso conscientemente organizado y con objetivos definidos, existe una diversidad de términos, consecuencia de la falta de rigor científico, que generan contradicciones y confusiones. El primer término que se utiliza, alrededor del cual la posición es unánime, es el de proceso, pero los complementarios son el centro de la polémica: pedagógico, educativo, de enseñanza, de aprendizaje, de enseñanza-aprendizaje y formativo. El debate se centra demás en si estos definen un solo proceso o si existen procesos con identidades distintas. Como en toda polémica existe al menos dos posiciones, para unos sí (González 2002) y para otros son varios, (Olivé y Finalés 2015).También se afirma que la Pedagogía es la ciencia que se ocupa de este proceso, o de estos procesos, según la filiación. El proyecto educativo, Gairin (2000), es la integración de acciones educativas, fundamentadas en la teoría y práctica pedagógica, concebidas y diseñadas sobre la base de una diagnóstico integral, contextualizado y sistemático, que se ejecutan y evalúan en una institución o grupo escolar, bajo la dirección científica de docentes y directivos, encaminadas a elevar la calidad de la educación para la formación integral de los estudiantes, cuyo protagonismo es decisivo, y en la que intervienen todos los componentes de la comunidad escolar. Según (Pla, et., al 2016) el proyecto educativo se convierte en estrategia de trabajo del colectivo pedagógico de año o de nivel, cuyos integrantes son los profesores, conducidos por un líder pedagógico, responsabilizados con la impartición de la docencia a los estudiantes de ese grupo.

Para Pla et al., (2010) el PE es "el sistema de interacciones del educando con la realidad fundamentalmente con las demás personas- organizadas en etapas y fases interdependientes constitutivas de una estrategia de la escuela u otra institución educativa que incluye el concurso de la familia y la comunidad y que se estructura sobre el fundamento de la teoría pedagógica- conforme a las necesidades sociales y contextuales derivadas estas en objetivos y contenidos precisos, concretados en una dinámica de base científica, flexible y creadora, dirigida por pedagogos para alcanzar como resultado la educación, la cual conduce al desarrollo de la personalidad". El PE es una categoría de máxima dimensión dentro la educación organizada institucionalmente con un carácter sistemático y profesional, es incluyente e integrador de todos las acciones educativas que se producen en tal contexto que puede ser de un centro educativo, de los medios de comunicación o de la familia, en cuyo marco tienen lugar otros procesos como 
el PEA. El primero debe ocurrir con una fundamentación pedagógica y el segundo con un sustento didáctico, aunque ambos tienen una relación tan dialéctica que se complementan. El PE es un proceso con propósito formativo. Se desarrolla en el marco de una institución educativa. Tiene un carácter sistémico, sistemático y profesional. Se sustenta en el sistema teórico de la Pedagogía.Integra la apropiación de una parte de la cultura, el conocimiento de una profesión, la capacidad para resolver los problemas de su actividad cotidiana. Incluye los rasgos de la personalidad, mediante los cuales se expresan los valores que los objetos y las personas tienen para el ser humano. Refleja la formación de las potencialidades funcionales del ser humano, sus facultades físicas, intelectuales y espirituales y tiene como objetivo la formación integral de la personalidad.

En ese proceso se interrelacionan objetivos y contenidos distintos. En primer término los objetivos y contenidos generales o transversales, vinculados con aspiraciones sociales acerca del ser humano que se necesita formar y los valores universales que deben revelar con su conducta, expresados en los fines de la educación y convertidos en ejes transversales de las actividades y formas organizativas del PE. En segundo lugar los objetivos y contenidos de los programas docentes vinculados a los sistemas de conocimientos, problemas y métodos de una o varias ciencias con arreglo didáctico en una asignatura. EI PE tiene manifestaciones en escenarios que no se reducen al aula, el taller o el laboratorio. En toda institución educativa se desarrollan programas culturales, se conmemoran efemérides históricas, se ejecutan actividades relacionadas con la educación formal, se realizan charlas informativas, se analizan y debaten realidades económicas y políticas comunitarias, nacionales, regionales internacionales y se emprenden proyectos educativos, todas propicias para el tratamiento de los contenidos formativos generales.

No es regularidad que el contenido de los ejes transversales adecue a los diferentes niveles de organización y sistematicidad del PE. Los contenidos de los programas docentes son objeto teórica y metodológicamente de la Didáctica y la teoría curricular vinculadas a la Pedagogía, pero se aprecia un vacío en el tratamiento de los contenidos generales desde la Pedagogía como ciencia para el PE. La ciencia que sustenta este proceso es la Pedagogía, cuyo objeto de estudio es: "el proceso educativo conscientemente organizado, sus leyes, principios, componentes y la dinámica de los contextos de actuación de los alumnos a través de las actividades que se desarrollan en la institución educativa, o en la familia y la comunidad bajo la orientación y control de la institución, y que incorporan, además de los objetivos y contenidos de los programas docentes, los objetivos y contenidos generales que se relacionan con lo artístico, lo deportivo, lo político y social extraclase, el trabajo intelectual independiente, el contacto con la naturaleza, contemplados como parte del sistema de interacciones que planifica la institución. (Pla et al., 2012)

\section{La Didáctica y el proceso de enseñanza-aprendizaje}

EI PEA es una forma de organizar el PE, ocurre dentro del mismo, su escenario más cotidiano es el aula, el taller, el laboratorio, el área de cultura física, o sea todos aquellos espacios concebidos para el tratamiento del contenido de una asignatura de la malla curricular: es el componente del PE institucional que de modo más sistémico organiza y estructura la enseñanza en relación con la manera que debe ocurrir el aprendizaje, a partir de la relación esencial que se da entre los fines de la educación (objetivos) y la precisión de los contenidos y de éstos con la dinámica (maestro, alumno, métodos, medios, formas, evaluación) a través de los cuales es posible lograr la educación vinculada de manera directa a un determinado contenido de las ciencias concretas, expresado en planes y programas de estudio (Pla et al., 2012)

Los fundamentos de este proceso le corresponden a la Didáctica, que según (Abreu et al., 2017) es una de las ciencias de la educación en pleno desarrollo. Está estrechamente vinculada con otras ciencias que intervienen en el proceso de enseñanza aprendizaje integrado e institucionalizado, especialmente con la Pedagogía, pero conserva sus particularidades y su esencia propia. Como ciencia orienta, socializa, integra y sistematiza en un cuerpo teórico en evolución ascendente, continua y sistemática, los resultados investigativos y de la experiencia acumulada en la práctica educativa, orientados a la exploración de la realidad del aula, a la detección, el estudio y la búsqueda de soluciones acertadas de los problemas que afectan e impiden el desarrollo óptimo, eficaz y eficiente del proceso de enseñanza-aprendizaje en su manifestación más amplia y contemporánea, que implica emocional y físicamente a profesores y estudiantes y los coloca en posición de éxito, en roles diferentes, pero con un propósito similar, a los primeros como guías, conductores del mismo y a los últimos como sujetos de su propio aprendizaje capaces de aprender el contenido de las asignaturas y los métodos para conseguirlo y de valorar críticamente las estrategias aplicadas para lograrlo, revela al método como parte del contenido, crea y desarrolla estructuras de participación que se sustentan en el diálogo y la retroalimentación, que facilitan la construcción y el desarrollo del aprendizaje, concebido, ejecutado y dirigido en el marco de instituciones educativas, para explicar, relacionar, demostrar y aplicar conocimientos necesarios para la vida práctica, en 
función de la formación integral de la personalidad, mediante el ascenso progresivo de la dependencia a la independencia autorregulada y a la capacidad de aprender por sí mismo durante toda la vida, en correspondencia con sus aspiraciones, sociales, grupales e individuales y el contexto, en un entorno histórico concreto.

\section{Los contenidos generales del proceso educativo}

En el PE intervienen de forma relacionada los contenidos generales y los contenidos de los programas docentes (específicos). Para Álvarez (1997) el contenido es: (...) la parte de la cultura seleccionada, con sentido pedagógico, para la formación integral del educando (...) se refiere a los conocimientos científicos: hechos, conceptos, teorías, enfoques, paradigmas. Incluye (...) modos (modelos, métodos) de pensamiento y actuación aceptados (...) por la sociedad para la comprensión efectiva de los saberes científicos, del dominio de las fuentes requeridas para la actividad y para la comunicación social; (...) las actitudes, normas, y valores, productos de la acción humana, (...) es reflejo de la ciencia y, de la sociedad en general, lleva implícito las potencialidades para que el hombre lo enriquezca, lo transforme y se transforme a sí mismo, (...) es una parte de la cultura que integra conocimientos, modos de pensar, actuar y sentir, y valores personales y sociales, que se seleccionan con criterios pedagógicos con el propósito de formar integralmente al educando.

En la concepción de la Pedagogía de la educación integral el contenido no es estático, se modifica con los resultados de la ciencia y las transformaciones sociales. Los resultados de la ciencia son objeto de conocimiento por sí mismos y son instrumentos para el aprendizaje de la problemática social. En los contenidos generales se incluyen los que las ciencias aportan acerca de temas relacionados con los problemas que la sociedad enfrenta en un momento históricamente concreto y que en ocasiones son resultado de estudios multidisciplinarios sobre una esfera de desarrollo social. Los contenidos generales, resultado de la cultura, están en las conclusiones de la ciencia, en las experiencias de los seres humanos, en sus relaciones y se integran en la formación de los educandos mediante conocimientos, intereses, orientaciones, actitudes y valores, en esferas como la salud, género y sexualidad, el medio ambiente, lo político ideológico, la orientación profesional y para el trabajo, la educación ética, estética, científico tecnológica, religiosa, la identidad cultural, la educación económica y jurídica, entre otras. Son influenciados por los procesos económicos, políticos, sociales, culturales y en su conjunto por la ideología que responde a una u otra clase. La problematización de los contenidos a partir de la influencia del contexto permite que el alumno los construya en relación con sus experiencias, en un proceso que incluye el aprendizaje individual y es determinado por el aprendizaje social.

El carácter contextual y problematizador de los contenidos generales influye en que estos no puedan ser seleccionados y secuenciados por organismos superiores del estado o el ministerio de educación. Se sugieren a partir de la formulación de los objetivos generales que orientan hacia los mismos y el planteamiento de temas que deben abordarse sobre la base de los resultados de la ciencia y los intereses de la sociedad. La universidad es la que debe seleccionar y secuenciar los mismos a partir de diversas fuentes, particularmente de resultados de investigaciones y experiencias con el trabajo en dichos temas. Esta característica diferencia a los contenidos generales del PE de los contenidos de los programas docentes que han sido seleccionados en otros niveles de educación y se enriquecen en la institución con las vivencias y experiencias sociales de la comunidad educativa y son objeto del PEA. Estos contenidos deben ser planteados en forma de temas generales a partir de la necesidad de atender a la ley pedagógica básica que expresa: "La relación, socialmente determinada, entre los fines del proceso educativo, la determinación del contenido y su dinámica interna en la educación de los alumnos". (Pla et al., 2012). Esta ley distingue cualitativamente la educación desde el PE, ya que revela un proceso conscientemente organizado y conducido, condicionado por las relaciones sociales predominantes en una época determinada. Los contenidos generales de dicho proceso también deben secuenciarse en los años o niveles académicos para lograr ciclos de profundidad y sistematicidad e impedir la repetición de su tratamiento. En la Educación Superior se utilizan estrategias curriculares que deben integrarse en una sola estrategia de carrera y en los proyectos educativos, lo cual también los diferencia de los contenidos específicos.

Anomalías en el tratamiento de los contenidos generales, como la tendencia a ofrecer orientaciones y plantearse objetivos generales para determinar las actividades y las formas organizativas, sin realizar la selección y secuenciación adecuada de los mismos, influyen en la falta de sistematicidad en el trabajo del $\mathrm{PE}$, pues los contenidos generales descontextualizados tienen limitada significación para los educadores y los estudiantes. Los contenidos generales se seleccionan en la institución educativa, a partir de los objetivos generales y sobre la base de temas propuestos por grupos de especialistas multidisciplinarios en los que participan pedagogos, para un nivel educativo determinado o para asignaturas del plan de estudio, sustentados en resultado investigativos. Demandan una secuencia para su tratamiento y la determinación de métodos educativos, de medios y formas organizativas dentro del PE. Tienen un carácter directriz para este proceso y la Pedagogía ofrece la forma metodológica de proceder para abordarlos. En ese proceso es 
determinante la participación de la comunidad educativa, no solo del educador y los educandos, pues mientras más participativo sea y más se relacione con los problemas del contexto, su significación será mayor para la educación integral de los estudiantes y garantizará una mejor contribución a la formación de los valores.

Álvarez (1997) plantea que el contenido es el medio fundamental de la formación del educando, es la concreción de lo que se quiere lograr en él, es el qué de la educación: qué aprendizajes, en qué sentido se desarrolla el individuo, a qué aspectos de la personalidad atender, qué tipo de proyecto de vida construir, qué relaciones tendrá con los demás, qué aspectos de la sociedad tienen que ver con el proceso de enseñanzaaprendizaje, está delimitado por el para qué, por el objetivo. La dirección del proceso, las finalidades, el enfoque que lleva las intenciones del proceso determinan el tipo, volumen y características del contenido. Los objetivos apuntan hacia la finalidad e intención del proceso; los contenidos llenan aquellos en términos de conocimientos, habilidades y actitudes. El contenido en función de los objetivos, orienta los métodos adecuados y, con ello, facilita la comprobación de lo logrado por los alumnos. Las actitudes constituyen el elemento más operativo de los contenidos generales, mediante ellas pueden irse formando los valores. Los contenidos generales se trabajan actualmente de formas diferentes, cada una de la cuales tiene sus características. Las actitudes son un componte indispensable de los contenidos generales.

Tabla 3: Formas en que se trabajan los contenidos en el proceso educativo

\begin{tabular}{|c|c|c|c|}
\hline Formas & La clase & $\begin{array}{l}\text { Programas directores, } \\
\text { ejes transversales y } \\
\text { estrategias curriculares }\end{array}$ & Las asignaturas \\
\hline Características & $\begin{array}{l}\text { Forma más común, } \\
\text { estudiada, controversial y } \\
\text { utilizada por la ciencia en } \\
\text { su integración a los } \\
\text { contenidos de las } \\
\text { asignaturas. El contenido } \\
\text { de la ciencia que nutre la } \\
\text { asignatura puede estar } \\
\text { relacionado directamente } \\
\text { con los temas o problemas } \\
\text { del contenido general o } \\
\text { reflejar aquellos que } \\
\text { intencionalmente permitan } \\
\text { asimilar los contenidos } \\
\text { generales seleccionados. } \\
\text { En la práctica educativa } \\
\text { hay una expresión muy } \\
\text { común y poco adecuada, } \\
\text { que es darles salida a } \\
\text { través de la clase a los } \\
\text { contenidos generales }\end{array}$ & $\begin{array}{l}\text { Selección y } \\
\text { secuenciación en los } \\
\text { programas directores - } \\
\text { ejes transversales según } \\
\text { la literatura occidental- } \\
\text { estrategias curriculares - } \\
\text { según la Educación } \\
\text { Superior Cubana. Esta } \\
\text { última denominación no } \\
\text { es compatible con el } \\
\text { cuerpo teórico de la } \\
\text { Pedagogía, que orientan } \\
\text { su tratamiento en todas } \\
\text { las asignaturas y otras } \\
\text { formas de organizar el } \\
\text { PE, en matutinos, actos, } \\
\text { círculos de interés, } \\
\text { sociedades científicas, } \\
\text { investigaciones, prácticas. }\end{array}$ & $\begin{array}{l}\text { Creación de asignaturas en el currículo } \\
\text { para tratar contenidos generales. Va en } \\
\text { detrimento del contenido de las ciencias } \\
\text { que se aborda en las asignaturas y del } \\
\text { tiempo que se le dedica, lo que afecta los } \\
\text { niveles de profundidad y de sistematicidad } \\
\text { en la manera de tratarlos. Tendencia } \\
\text { actual. Práctica que generalizada es } \\
\text { negativa. Colma la malla curricular de } \\
\text { asignaturas y se desaprovechan otras } \\
\text { formas más relacionadas con intereses y } \\
\text { motivaciones de los alumnos. Todo lo que } \\
\text { se incluye en una asignatura no es } \\
\text { garantía de una mejor educación, a veces } \\
\text { genera un efecto contrario. Los círculos } \\
\text { de interés, los espacios abiertos de } \\
\text { debate, las actividades extradocentes, } \\
\text { laborales, deportivas, políticas, culturales, } \\
\text { recreativas y los proyectos comunitarios, } \\
\text { pueden tener un efecto más educativo } \\
\text { que una asignatura del currículo. }\end{array}$ \\
\hline
\end{tabular}

Tabla 4: Las actitudes según Gairin (2013)

\begin{tabular}{|l|l|}
\hline \multicolumn{1}{|c|}{ Actitudes } & \multicolumn{1}{c|}{ Características } \\
\hline $\begin{array}{l}\text { Constructos conductuales multidimensionales, } \\
\text { suponen una predisposición a la acción } \\
\text { relativamente estable y susceptible de formación } \\
\text { y cambio en lo que interactúan entre sí tres } \\
\begin{array}{l}\text { componentes: el cognitivo, el afectivo y el } \\
\text { comportamental. }\end{array}\end{array}$ & $\begin{array}{l}\text { Disposición a la acción. Se forman y pueden modificarse, aunque se } \\
\text { consideran una disposición relativamente estable. Principios de } \\
\text { organización mental en relacionados con un objeto o situación, que } \\
\text { se establece con arreglo a una polaridad afectiva. Tienen un } \\
\text { carácter multidireccional pues integran diversos componentes a } \\
\text { través de los que se analiza la conducta. }\end{array}$ \\
\hline
\end{tabular}

Los objetivos generales se relacionan fundamentalmente con las actitudes en función de lograr los valores a más largo plazo, por tanto los contenidos generales, determinados por los objetivos generales, se revelan en forma de actitudes. Esta característica distingue a los contenidos generales del PE de los contenidos de los programas docentes. Los contenidos generales son "un componente del proceso educativo que integra aspectos cognitivos, afectivos y comportamientos relacionados con actitudes y valores sobre problemáticas multidisciplinarias de la educación integral de los estudiantes, están presente en las formas organizativas y actividades del PE, incluyendo el PEA, se estructuran a partir de los problemas sociales en diferentes esferas del desarrollo y de la fundamentación en las ciencias y se enriquecen sistemáticamente con las 
problemáticas del contexto más inmediato, además de las vivencias, motivos e intereses de los estudiantes y adquieren determinado grado de importancia de acuerdo al contexto y características particulares del proceso educativo" (Pla et al, 2012).

La relación que en el PEA se establece entre los conocimientos, habilidades, valores, experiencias de la actividad, como componentes del contenido de los programas docentes, en el PE se manifiesta entre lo cognitivo (conocimiento-habilidad), incluyendo el aspecto histórico y tradicional identitario, sobre la actitud, los motivos personales respecto a esa actitud y las características de los comportamientos en concordancia con la aspiración social, siendo esencial lo afectivo para su asimilación y el comportamiento del indicador externo de la asimilación de los contenidos generales. El fin del PE es la contribución a la formación de los valores. Los contenidos generales en su concreción didáctica en la asignatura deben transformar las actitudes que forman los valores en los diferentes niveles educativos, por tanto, los contenidos generales y los contenidos de las asignaturas debían coincidir en la formación de actitudes, y como consecuencia, la formación de valores. Sin embargo, la necesidad de determinar contenidos generales del PE significa que no es suficiente lo que las asignaturas aportan a la educación integral de los alumnos, por tanto, los contenidos generales tienen el propósito de trabajar directamente en las actitudes, lo cual los distingue. "Los componentes cognitivos, afectivos y comportamentales son congruentes entre sí, aunque es el afectivo de mayor importancia en la configuración de las actitudes, como opinan Fishbein y Azjen (2000). El PE integra tres componentes, el cognitivo, el afectivo y el conductual.

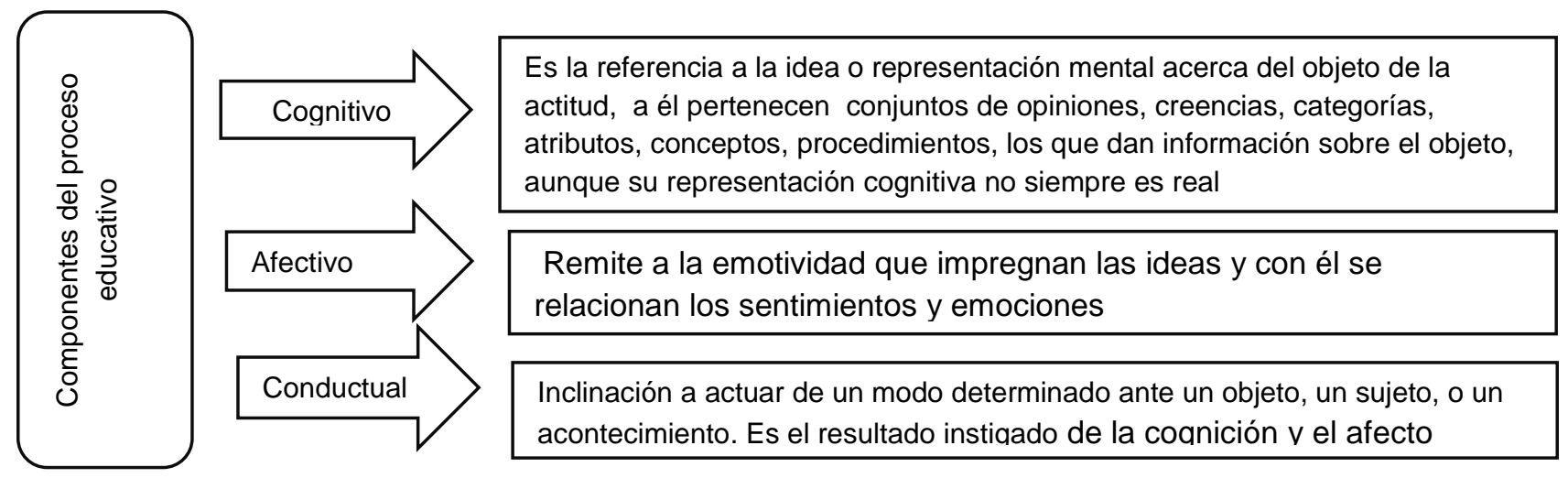

Fig. 1: Representación de los componentes del proceso educativo

El valor es la convicción perdurable que evidencia que un modo específico de conducta o estado final de existencia, es personal o socialmente preferible a un modo de conducta o estado final de existencia opuesto o contrario. Los contenidos del PE tienen en los valores sus generalizaciones más significativas, ellos constituyen el núcleo de los contenidos de dicho proceso e incluyen los ideológicos. La ideología es el nivel superior de la conciencia social y la moral es una forma de esa conciencia. Los valores se relacionan con las actitudes ya que son integradores de actitudes. De acuerdo al valor o valores que asume una persona, así serán sus actitudes en la mayoría de las situaciones en las que se ve involucrado. Los valores son un sistema. Son creaciones sociales que una vez personalizados y convertidos en cualidades de la personalidad se expresan en las actitudes y conductas de las personas para incluir en los contenidos del PE a esas manifestaciones de los valores. La actitud es una postura de la personalidad ante determinado aspecto de la realidad, que se hace estable y forma parte de las formaciones personológicas. Las actitudes son la aspiración de comportamiento de una persona, que en ocasiones no se corresponden con la conducta observada (contradicción entre actitud y conducta) porque al sujeto le pueden faltar otras formaciones como: hábitos y habilidades. Este tipo de razonamiento es decisivo para guiar el desarrollo del educando desde los logros actuales hasta los logros futuros. Pueden ser contenidos del PE actitudes y conductas como: uso correcto del uniforme escolar, el aspecto personal, cuidado de la limpieza y la organización, ahorro de recursos, trato respetuoso a los demás, delicadeza al hablar, la caballerosidad, el sentido del respeto a sí mismo y los demás, asistencia sistemática a clases y demás actividades, puntualidad, cumplimiento del trabajo independiente y los deberes, coherencia entre lo que se dice y lo que se hace y entre lo que se exige y lo que se aporta.

\section{Formación de valores y los contenidos generales}

La formación de o en valores ha sido tratada por autores como Ajzen y Fishbein (2000), Aranda et., al (2005), Martínez (2006), Casares et., al (2010), Osuna et., al (2012) y Vidal y Araña (2014). En este caso se proponen algunos de los valores más demandados por la sociedad contemporánea y sus definiciones, cuya formación fue potenciada mediante el tratamiento de los contenidos generales desde la Pedagogía. 
Dignidad: el respeto a sí mismo, a la patria y a la humanidad. Actuar con libertad y con capacidad para desarrollar actividades diversas. Sentir orgullo por las acciones que se realizan en defensa de los intereses de la sociedad. Merecer ser respetado por ser consecuente con los principios y por lograr correspondencia entre lo que se piensa, proclama y hace. Patriotismo: considerar a la patria como madre cultural. Identificación, pertenencia y amor a la nación y a sus habitantes, a la naturaleza, la sociedad, la cultura y la historia. Compromiso con sus compatriotas. No se limita a la educación patriótica, ni a la adoración de personalidades, incluye la importancia de las masas populares en los procesos históricos. Humanismo: amor a los seres humanos y preocupación por el desarrollo pleno de todos sobre la base de la justicia. Solidaridad: comprometerse en idea y acción con el bienestar de los otros: en la familia, la escuela, los colectivos laborales, la nación y habitantes de otros países. Estar atento a toda la humanidad. Fortalecer el espíritu de colaboración y de trabajo en equipo. Apreciar en alto grado el sentido de compañerismo y compartir los recursos, en aras de potenciar el conocimiento. Desarrollar una cultura que privilegia el trabajo integrado en red, la consulta colectiva, el diálogo y debate para la identificación de los problemas y la unidad de acción en la selección de posibles alternativas de solución. Identificarse con el sentido de justicia social, equidad e internacionalismo, ante las causas nobles que pueden lograr un mundo mejor, de paz e igualdad. Actuar como buen ser humano, experimentar felicidad cuando contribuye con la felicidad de los demás.

Otros valores son. Laboriosidad: condición necesaria para la eficacia de la educación considerando el protagonismo del trabajo en la formación del ser humano y la dependencia entre sociedad y trabajo. Amor a los trabajadores y reconocimiento a los aportes de la actividad laboral productiva o de servicios a la formación moral. Honradez: rectitud e integridad en todos los ámbitos de la vida. Necesidad de vivir de su propio trabajo y esfuerzo. Actuar de manera sincera, sencilla y veraz. Expresar juicios críticos y reconocer errores en tiempo, lugar y forma adecuada para contribuir al bien propio, colectivo y de la sociedad. Lograr armonía entre el pensamiento, el discurso y la acción. Honestidad: actuar de manera sincera, sencilla y veraz. Ser decente, decoroso y recatado. Defender la verdad y la justicia. Actuar con integridad. Respetar las buenas costumbres, los principios morales y los bienes ajenos. Lograr armonía entre el pensamiento, el discurso y la acción. Actuar con transparencia ante lo justo en el colectivo. Ser sincero con apego a la verdad y exigir lo mismo de los demás. Ser ejemplo en el cumplimiento de los deberes. Justicia social: respeto a la igualdad social que se expresa en que los seres humanos sean acreedores de los mismos derechos y oportunidades, sin discriminación por diferencias de origen, edad, sexo, ocupación social, desarrollo físico, mental, cultural, color de piel, credo y de cualquier otra índole. Responsabilidad: cumplimiento del compromiso contraído ante sí mismo, la familia, el colectivo y la sociedad. Posibilitar la creación de un clima de autodisciplina en el desempeño de misiones en las actividades cotidianas. Desplegar todas las potencialidades en la conquista del entorno, con audacia responsable. Gratitud: ser agradecido. Sentir estima y reconocimiento por quienes han contribuido con su formación, desarrollo, la definición de un proyecto de vida adecuado y el logro de sus objetivos y corresponderles con su actuación y tolerancia. Reconocer y respetar la diversidad en toda su dimensión opiniones, ideas, pensamiento, actitudes y acciones de las demás personas, aunque no coincidan con las propias.

\section{Exigencias pedagógicas para el tratamiento de los contenidos generales}

El PE propicia situaciones en las que los alumnos manifiesten sus actitudes. La acumulación de experiencias y la confrontación social con el grupo u otras estructuras de la comunidad o de los medios de comunicación, van definiendo valores en relación con la significación individual y socialmente positiva que tienen los procesos, las personas, las cosas, que se manifiestan en contradicción con otras maneras de actuar respecto a los mismos objetos. El PE demanda condiciones coincidentes con los contenidos generales: transformar en conocimientos y habilidades las informaciones acerca de las actitudes y valores objeto de análisis, vivir las experiencias positivas o negativas ante el objeto de las actitudes y contraponer los conocimientos, habilidades y las vivencias con las normas socialmente aprobadas para una sociedad históricamente determinada, siempre desde el enfoque ideológico de las clases sociales. En el proyecto educativo se necesita crear las condiciones para que puedan ser asimilados todos los componentes del contenido, no basta con informar cómo la sociedad quiere que sean los valores de los educandos, es imprescindible que se practiquen actitudes y confronten socialmente los comportamientos.

La formación de actitudes parte del contenido motivo de estudio, en el cual se selecciona la parte que será objeto de la actitud a formar y que deberá reunir las características afectivas y motivacionales. Es necesario que se delimiten las conductas correspondientes y significativas de dicha actitud que serán vivenciadas por los alumnos, en situaciones de aprendizaje. El conocimiento que el estudiante va aprendiendo, se presenta en la actividad -que requiere una experiencia vivenciada- en la que ejercitará habilidades y formará actitudes. Si no existe situación afectiva que forme actitud, no se aprenden conocimientos y habilidades y si no hay ejercitación de habilidades, no se asimilan conocimientos y no se forman actitudes.

La selección y estructuración de los contenidos generales, que incluyen los conocimientos y habilidades sobre las actitudes, las vivencias afectivas y los comportamientos, exige de la labor creativa de los 
educadores. Para la actitud sobre la disposición al trabajo socialmente útil se necesita precisar en ese contenido información relacionada con el conocimiento de lo que significa estar dispuesto para el trabajo, saber cuál es el tipo de trabajo que se realizará, las habilidades para realizarlo, qué importancia tiene, social e individualmente y las condiciones para realizarlo. Es necesario precisar los motivos individuales, los intereses, lo afectivo respecto a esa actitud y cuáles serán los comportamientos que se socialmente son aceptados respecto a dicha disposición al trabajo. La selección y estructuración de la actitud como contenido de la educación exige que el educador cree las condiciones para transitar metodológicamente por los conocimientos, las habilidades, las vivencias afectivas y los comportamientos, su coincidencia con las actitudes sociales que se observen del resto de las personas, la repetición reiterada y en nuevas condiciones del tratamiento de dicho contenido y debe influir en la asimilación de la actitud y en la conformación del valor relacionado con el amor al trabajo.

Determinación de los contenidos generales necesarios para la formación profesional de los estudiantes y que trascienden los contenidos de las asignaturas

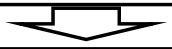

Determinación de los problemas sociales e individuales cuya solución se asocia al dominio de dichos contenidos

y la participación de los estudiantes

Selección de los contenidos generales para el proyecto educativo de año o nivel, según diagnóstico, contexto y necesidades de la formación del profesional con la particinación de los intearantes de la comunidad educativa

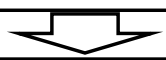

Establecimiento de las responsabilidades individuales y grupales en el tratamiento de los contenidos seleccionados

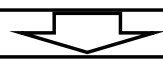

Diseño de mini proyectos por los estudiantes para la solución de problemas comunitarios y/o profesionales, mediante el tratamiento de los contenidos generales

Planteamiento ante el colectivo de lo que realizará cada cual y cómo se integra al proyecto del grupo

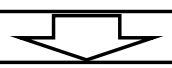

Seguimiento sistemático del cumplimiento del diseño de las actividades, individuales y colectivas

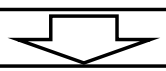

Control y evaluación individual y colectiva del cumplimiento de las actividades y los resultados

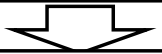

Estimulación de los logros y evaluar la calidad y factibilidad del sistema de acciones ejecutado

Fig. 2: Pasos metodológicos para el tratamiento de los contenidos generales

\section{CONCLUSIONES}

De los resultados que se lograron, se derivan las siguientes conclusiones: 1) Los contenidos generales son objeto de atención de la Pedagogía en sus precisiones teóricas y metodológicas. 2) Pueden organizarse de forma lineal, por ciclos o por temas, para evitar repeticiones, según el enfoque humanista y social de la Pedagogía. 3) EI PE es su forma de organización y el proyecto educativo su concreción. 4) Los contenidos generales son un potencial para la formación de valores de los estudiantes.

\section{REFERENCIAS}

Abreu, O., Gallegos, M., Jácome, J. y Martínez, R. La Didáctica: Epistemología y Definición en la Facultad de Ciencias Administrativas y Económicas. DOI. 10.4067/S0718-50062017000300009. Revista Formación Universitaria, 10 (3), 81-92, (2017)

Abreu, O., Naranjo, M., Rhea, B., y Gallegos, M. Modelo Didáctico para la Facultad de Ciencias Administrativas y Económicas de la Universidad Técnica del Norte en Ecuador. DOI: 10.4067/S071850062016000400002. Formación Universitaria, 9 (4), 03-10 (2016) 
Ajzen, I., Fishbein, M. Attitudes and the Attitude-Behavior Relation: Reasoned and Automatic Processes. DOI.10.1080/14792779943000116. European Review of Social Psychology, 11, 1-33 (2000)

Álvarez, C. La Pedagogía como Ciencia. Epistemología de la Educación. Pueblo y Educación, 1-164, La Habana, Cuba (1999)

Álvarez, R.M. Hacia un Curriculum Integral y Contextualizado. Académica, 1-138, La Habana, Cuba (1997)

Aranda, J.S. y Salgado, E. La formación de Valores en el Ser Humano Innovación. ISSN: 1665-2673. Revista Educativa, 5 (28), 33-43 (2005)

Bolívar, A. Conocimiento didáctico del contenido y didácticas específicas. Profesorado. Revista de currículum y formación del profesorado, ISSN-e: 1138-414X, 9, 2 (2005)

Casares, P.M., Carmona, G., Martínez-Rodríguez, F.M. Valores profesionales en la formación universitaria. E-ISSN: 1607-4041 REDIE. Revista Electrónica de Investigación Educativa, 12(1), 1-15 (2010)

Díaz, J.A. Conocimiento didáctico del contenido para la enseñanza de la naturaleza de la ciencia (i): el Marco Teórico. E-ISSN: 1697-011X. Revista Eureka sobre Enseñanza y Divulgación de las Ciencias, 6(1), 21-46 (2009)

Gairin, J. El Proyecto Educativo y el Desarrollo del Currículum. ISBN: 84-7827-233-X. Laboratorio Educativo, 3-32 (2000)

Gairin, J. y Muñoz J.L. La organización Escolar Como Campo de Estudio e Investigación. ISSN 1137-3636. Revista Anthropos: Huellas del Conocimiento, 238, 11-27 (2013)

González, A. El Proceso de Enseñanza-Aprendizaje ¿Agente del cambio educativo? En: Nociones de Sociología, Psicología y Pedagogía. Editorial Pueblo y Educación, 1-177 (2002)

Martín, R., Fernández, P., González, M. y Oliva, A. El Dominio de los Contenidos Escolares: competencia Profesional y Formación Inicial de Maestros. ISSN: 0034-8082. Revista de Educación, 360, 363-387 (2013)

Martínez, M. Formación Para la Ciudadanía y Educación Superior. ISSN: 1022-6508. Revista Iberoamericana de Educación, 42, 85-102 (2006)

Medina, J.L. y Jarauta, B. Análisis del conocimiento didáctico del contenido de tres profesores universitarios. DOI: 10-4438/1988-592X-RE-2011-360-131. Revista de Educación, 360, 600-623 (2013)

Olivé, M.A. y Finalés, R. La pedagogía como ciencia. Hacia una definición de su objeto de estudio. ISSN: 1989-4155. Revista Atlante: Cuadernos de Educación y Desarrollo, 1-8, (2015)

Osuna, S. Laso, M. y Aparici, R. Valores de la formación universitaria de los comunicadores en la sociedad digital: más allá del aprendizaje tecnológico, hacia un modelo educomunicativo. ISSN: 1605-4806. Revista Razón y Palabra, 81, 1-33 (2013)

Pla, R. y otros 16 autores. Una concepción de la Pedagogía como ciencia desde un enfoque histórico cultural. $1^{a}$ Ed., Pueblo y Educación, 1-79, La Habana, Cuba (2010)

Pla, R., Ramos, J. La categoría contenido en el proyecto educativo para la formación integral de los profesionales. Revista "Actualidades Investigativas en Educación". ISSN: 1409-4703, 16(3), 1-24 (2016)

Vidal, M. y Araña, B. Formación en Valores. ISSN 0864-214. Revista Educ. Med. Super., 28 (1), 175-186 (2014) 